This item was submitted to Loughborough's Research Repository by the author.

Items in Figshare are protected by copyright, with all rights reserved, unless otherwise indicated.

\title{
Single-shot profilometry of rough surfaces using hyperspectral interferometry
}

PLEASE CITE THE PUBLISHED VERSION

http://dx.doi.org/10.1364/OL.37.000350

PUBLISHER

(C) Optical Society of America

VERSION

VoR (Version of Record)

LICENCE

CC BY-NC-ND 4.0

REPOSITORY RECORD

Widjanarko, Taufiq, Jonathan M. Huntley, and Pablo D. Ruiz. 2019. "Single-shot Profilometry of Rough Surfaces Using Hyperspectral Interferometry”. figshare. https://hdl.handle.net/2134/13242. 
This item was submitted to Loughborough's Institutional Repository (https://dspace.lboro.ac.uk/) by the author and is made available under the following Creative Commons Licence conditions.

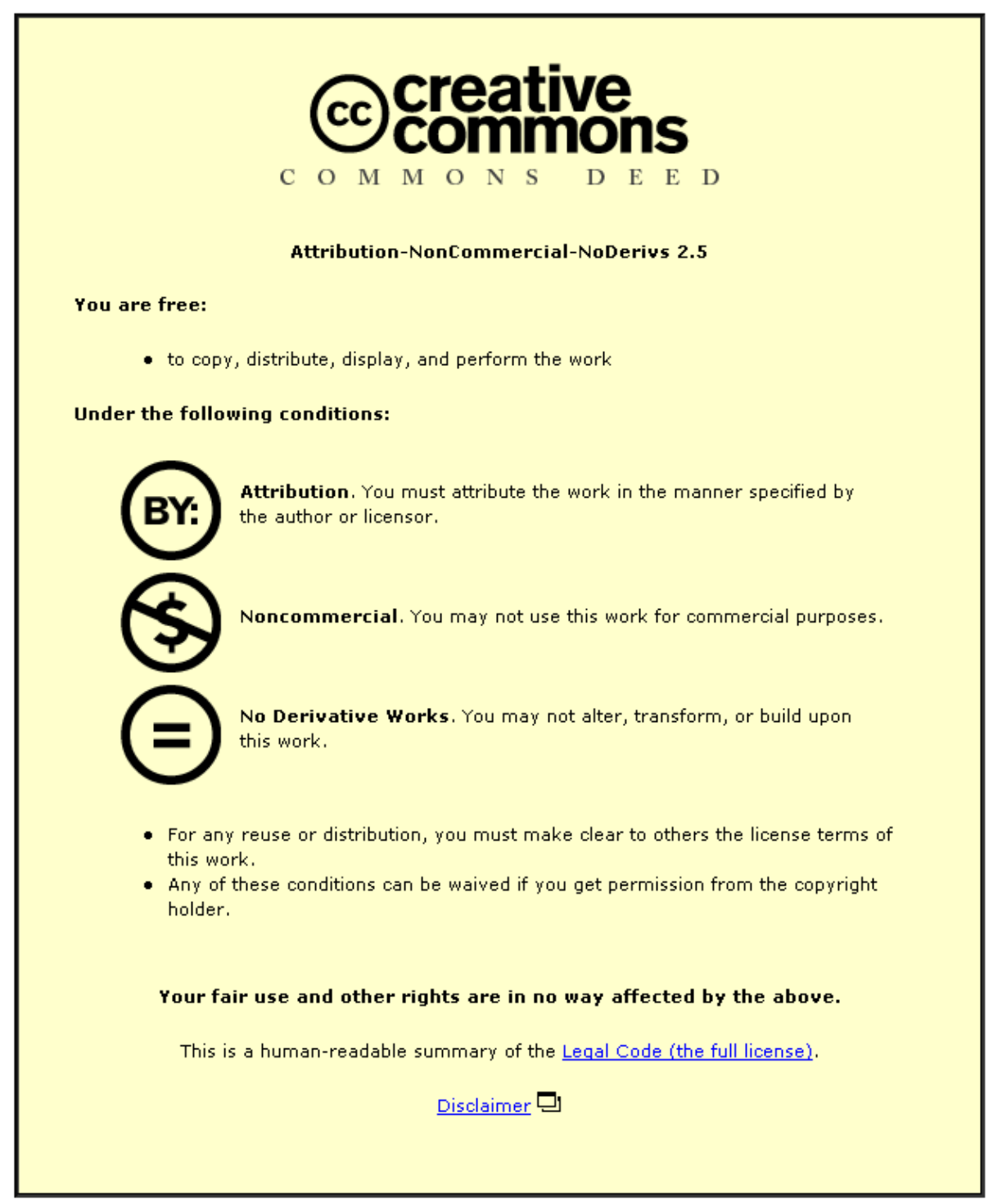

For the full text of this licence, please go to: http://creativecommons.org/licenses/by-nc-nd/2.5/ 


\title{
Single-shot profilometry of rough surfaces using hyperspectral interferometry
}

\author{
Taufiq Widjanarko ${ }^{1,2}$, Jonathan M. Huntley, ${ }^{1, *}$, and Pablo D. Ruiz ${ }^{1}$ \\ ${ }^{1}$ Wolfson School of Mechanical and Manufacturing Engineering, Loughborough University, Loughborough LE11 3TU, UK \\ ${ }^{2}$ Current address: EPSRC Center in Through-life Engineering Services, School of Applied Sciences, \\ Cranfield University, Cranfield MK43 OAL, UK \\ ${ }^{*}$ Corresponding author: j.m.huntley@lboro.ac.uk
}

Received October 25, 2011; accepted November 14, 2011;

posted December 2, 2011 (Doc. ID 156789); published January 20, 2012

\begin{abstract}
The combination of white light interferometry with hyperspectral imaging ("hyperspectral interferometry") is a recently proposed technique for single-shot measurement of 3D surface profiles. We consider for the first time its application to speckled wavefronts from optically rough surfaces. The intensity versus wavenumber signal at each pixel provides unambiguous range information despite the speckle-induced random phase shifts. Experimental results with samples undergoing controlled rigid body translation demonstrate a measurement repeatability of $460 \mathrm{~nm}$ for a bandwidth of approximately $30 \mathrm{~nm}$. Potential applications include roughness measurement and coordinate measurement machine probes where rapid data acquisition in noncooperative environments is essential. (c) 2012 Optical Society of America

OCIS codes: $120.3180,120.6660$.
\end{abstract}

Interferometry with monochromatic light has been used for many years to measure the surface profiles of optically smooth components such as lenses or silicon wafers. Subnanometer accuracy is possible when combined with phase shifting techniques. Phase unwrapping (and hence height measurement) errors occur, however, on objects with slopes sufficiently steep (e.g., due to discontinuities or surface roughness) for the phase gradient to exceed the Nyquist limit of $\pm \pi$ per pixel. This ambiguity problem can be remedied by using multiple wavelengths, where at least two approaches may be used. The first, on which many commercial profilometers are based, is known as scanning white light interferometry [1]. In this type of interferometer the object is moved while keeping the reference beam stationary, and the point of maximum fringe modulation is recorded on a pixelwise basis. In the second approach known as wavelength scanning interferometry [2, 3 ], both interferometer arms remain fixed and the wavelength is scanned over time. Both approaches require the recording of typically hundreds of images or more, during which time the interferometer and sample must remain stable. It is desirable therefore to consider how to measure a surface profile with single-shot image acquisition, thus minimizing the effects of environmental disturbance.

Such a feature is provided by an alternative broadband interferometric approach called hyperspectral interferometry (HSI) [4]. HSI uses a hyperspectral imager to spatially separate a set of narrowband interferograms from a single white light interferogram. Unambiguous measurement of two-dimensional (2D) optical path distributions can thus be obtained in a single shot. The 2D nature of the measurements distinguishes the technique from spectral optical coherence tomography, which provides only onedimensional (1D) distributions. A prototype HSI system and sample profiles from a smooth specularly reflecting step height object have been reported in [4]. In the current Letter we consider the response of the system to microscopically rough surfaces such as machined metal. Such surfaces give rise to speckled narrowband interfer- ograms where no continuous interference fringes are visible, unlike the smooth wavefront interferometer considered previously in [4].

The optical setup is shown in Fig. 1. Light from a broadband superluminescent LED source $\mathrm{LS}_{1}$ (S840-HP-I Superlum Diodes Ltd, Moscow; $15 \mathrm{~mW}$ optical power; center wavelength $840 \mathrm{~nm}$; bandwidth $50 \mathrm{~nm}$ ) is launched into an optical fiber. Light from narrowband HeNe laser source $\mathrm{LS}_{2}$ (wavelength $632.8 \mathrm{~nm}$ ), which is combined with that from $\mathrm{LS}_{1}$ using fiber coupler $\mathrm{FC}$, assists in the alignment of the setup.

On exiting the fiber, the light is collimated by lens $\mathrm{L}_{1}$ and spectrally filtered to produce a frequency comb by etalon E, which has free spectral range (FSR) of $0.5 \mathrm{~nm}$ at $840 \mathrm{~nm}$ and finesse $>15$. The filtered light subsequently enters the Linnik interferometer comprising beamsplitter $\mathrm{BS}_{1}$, lens $\mathrm{L}_{2}$, reference mirror $\mathrm{RM}$, and object lens $L_{3}$. The rough surface sample $S$ is mounted on

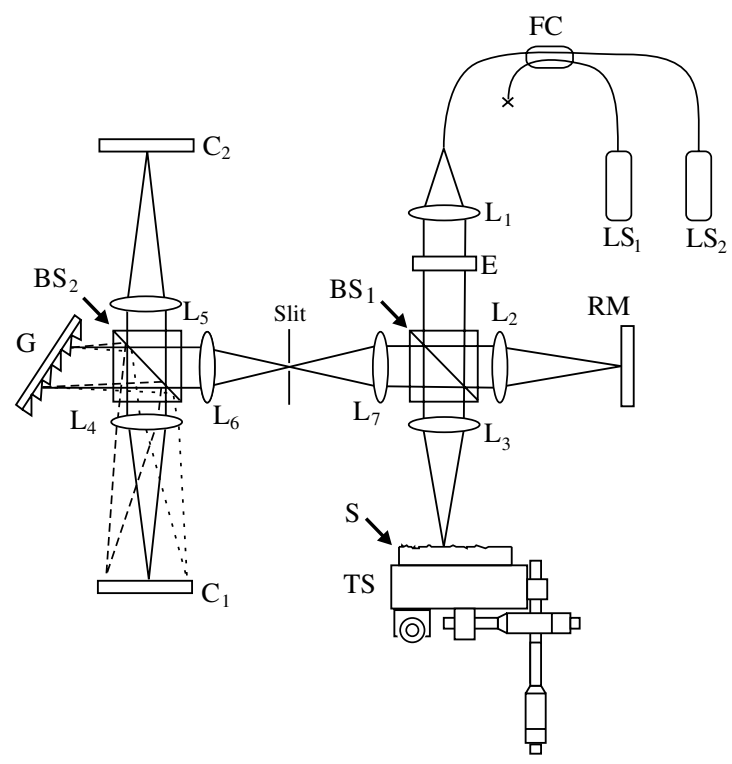

Fig. 1. Hyperspectral interferometry setup. 
translation stage TS. The scattered and reflected light from $\mathrm{S}$ and $\mathrm{RM}$, respectively, recombine at $\mathrm{BS}_{1}$ and travel to the hyperspectral imaging block of the system consisting of beamsplitter $\mathrm{BS}_{2}$, lenses $\mathrm{L}_{4}$ and $\mathrm{L}_{5}$, and grating $\mathrm{G}$. Cameras $\mathrm{C}_{1}$ and $\mathrm{C}_{2}$ record, respectively, the spatially separated narrowband interferograms and the broadband interferogram. The focal lengths of lenses $\mathrm{L}_{3}$ and $\mathrm{L}_{4}$ (100 and $150 \mathrm{~mm}$, respectively) provide an image magnification of $1.5 \times$ between $S$ and $C_{1}$. In order to suppress the crosstalk between two adjacent spectral images, a slit in conjunction with lenses $\mathrm{L}_{6}$ and $\mathrm{L}_{7}$ is used as a field stop. The sample $\mathrm{S}$ was a surface grinding roughness standard from Rubert \& Company Ltd, Cheshire, UK, with average roughness $\mathrm{Ra}$ of $3.2 \mu \mathrm{m}$.

An example of a set of 64 narrowband images recorded by camera $\mathrm{C}_{1}$ is shown in Fig. 2 , with the inset showing four such images enlarged by $10 \times$. The image was taken with the object arm only, i.e., with no reference wave. Without etalon $\mathrm{E}$ the grating will smear the images across the horizontal axis of $\mathrm{C}_{1}$. The presence of the etalon results in a set of replicated images taken at equally spaced wavenumbers corresponding to the peaks of the etalon's spectral transmission comb. The speckle pattern produced by the rough surface is clearly visible.

The images produced by simultaneous illumination by object and reference arms were later stacked to create a hyperspectral image volume. Each image has a size of $19 \times 19$ pixels, which corresponds to a field of view of $81.7 \times 81.7 \mu \mathrm{m}^{2}$. Techniques to register the images have been presented in [4]. The intensity of a pixel is denoted $I(x, y, k)$, where $x, y$ are the spatial coordinates within each image, and $k$ is the wavenumber. Three sample pixels $p_{j}(j=1,2,3)$ are shown in Fig. 3(a), and corresponding signals $I\left(x_{j}, y_{j}, k\right)$ in Fig. $3(\mathrm{~b})$ with vertical offsets to clarify the presentation. The spectral pedestal function has been removed, to eliminate low frequency terms from the subsequent 1D Fourier transforms along $k$ [4].

A vertical dashed line helps identify the intensity values of the three pixels for a selected image index (in this

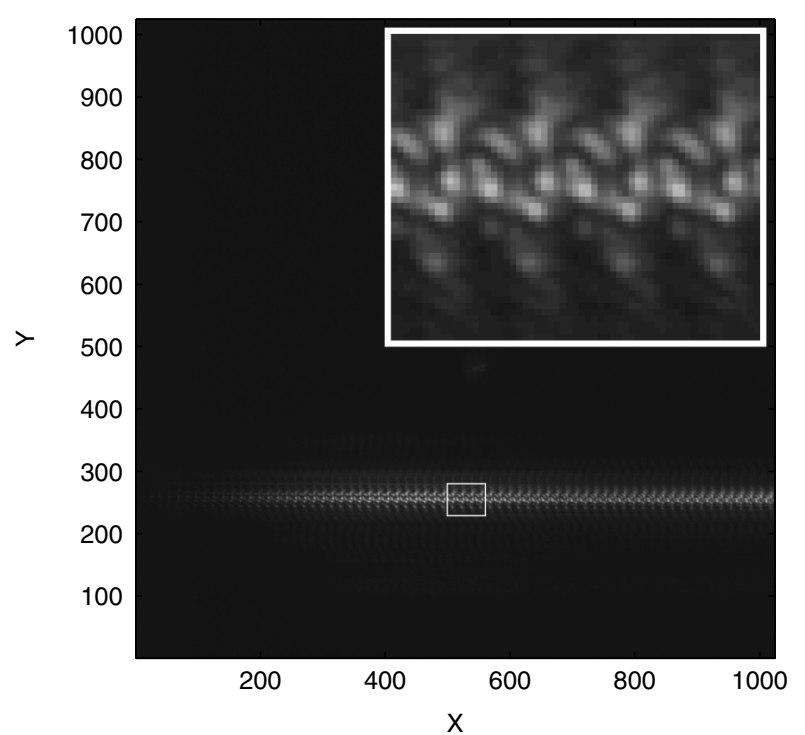

Fig. 2. Hyperspectral image set of the sample (object arm only).

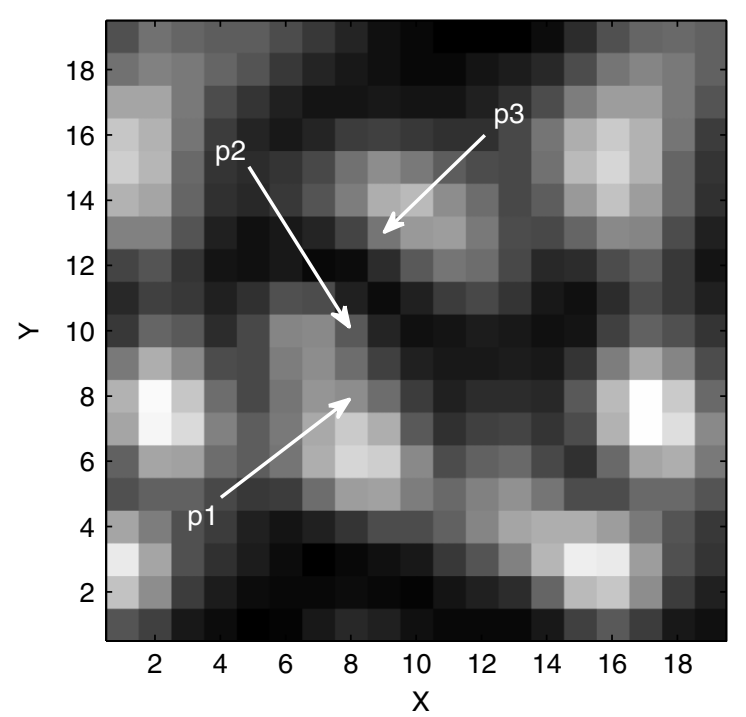

(a)

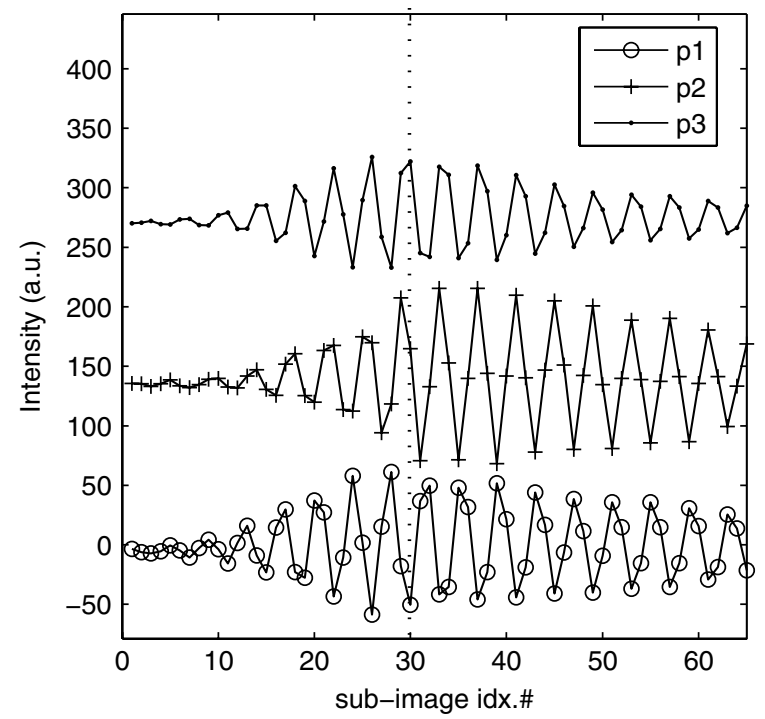

(b)

Fig. 3. (a) Image No. 30 from Fig. 2 with three selected pixels, and (b) the corresponding interference signals.

example No. 30). Since image index equates to a single $k$ value, the three intensities are equivalent to the signals that would be obtained by traditional speckle interferometry with monochromatic light. The random surface microstructure causes random phase offsets between pixels, which make depth measurement impossible in such a case even with phase shifting. HSI on the other hand uses the frequency of the signals $I\left(x_{j}, y_{j}, k\right)$ to encode depth, and this is unaffected by the random phase shifts seen in the signals of Fig. 3(b). 1D Fourier transformation along $k$, followed by location of the peak on a pixel by pixel basis, provides a map of surface height $h(x, y)$ [4].

A significant difference between speckled and smooth wavefronts is the presence of zero amplitude points. An additional spatial "speckle averaging" operation was needed to provide useful data at pixels whose object beam amplitude is nearly zero. This was implemented as the following local weighted smoothing operation 


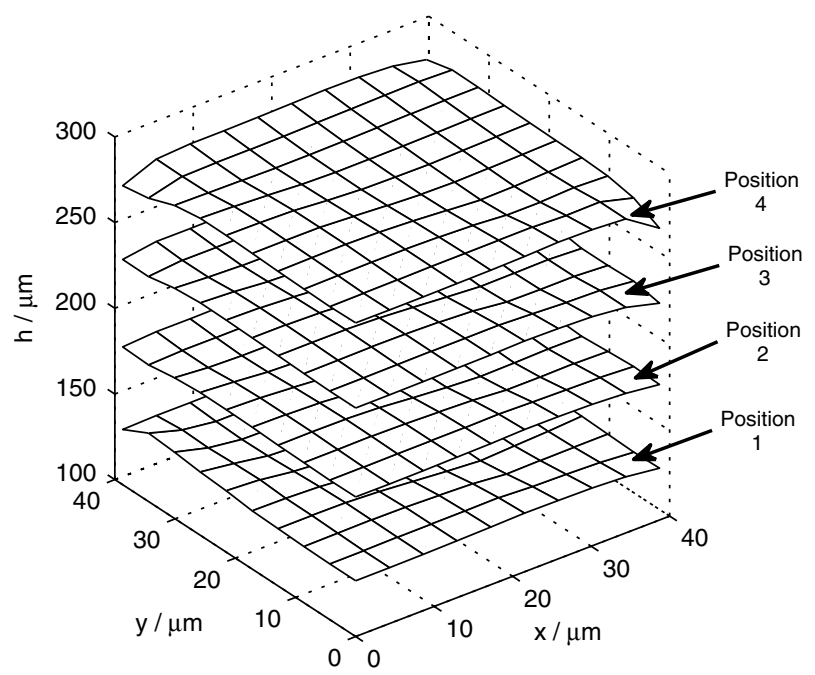

Fig. 4. Set of reconstructed surface height maps taken at four consecutive stage positions, each separated by $50 \mu \mathrm{m}$.

$$
h_{S}(x, y)=\frac{\left(\left|H_{p}(x, y)\right|^{2} h(x, y)\right) \otimes o(x, y)}{\left|H_{p}(x, y)\right|^{2} \bigotimes o(x, y)}
$$

where $h_{S}(x, y)$ is the smoothed height map, $\otimes$ denotes 2D convolution, $o(x, y)$ is the smoothing kernel (in this case a $4 \times 4$ matrix with each element equal to one), and $\left|H_{p}(x, y)\right|$ is the magnitude of the signal peak in the Fourier transform of $I(x, y, k)$.

To assess the system performance, the sample was moved axially in $50 \mu \mathrm{m}$ steps using the translation stage. The central region of the height maps from four known stage positions is shown in Fig. 4. The experimental data show the average distance between height maps is $50.16 \mu \mathrm{m}$, which is (to within the uncertainty of the stage) the same as the prescribed $50 \mu \mathrm{m}$ position increments.

The reconstructed height maps in Fig. 4 were further analyzed by subtracting the mean value from each, allowing the measured height fluctuations to be seen on an expanded vertical scale. The results are shown in Fig. 5 where the height fluctuations cover the range (with respect to the average value) $-3 \mu \mathrm{m}$ to $1 \mu \mathrm{m}$. Qualitatively the fluctuation distributions can be seen to be relatively consistent from one stage position to the other. This can be quantified through the rms difference between each distribution and the average of the other 3 , excluding a one-pixel wide border around each image. This gave a value of $460 \mathrm{~nm}$, which can be interpreted as an estimate of the repeatability of the system.

In conclusion, we have demonstrated how HSI can be used to measure 3D profiles of rough surfaces in a single shot. Unlike the case of smooth wavefront HSI, the
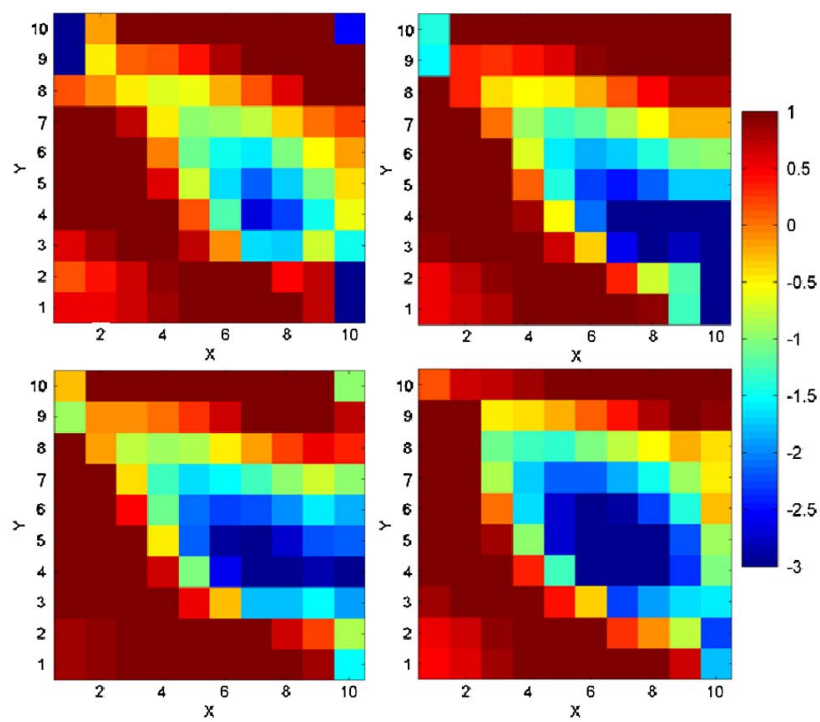

Fig. 5. (Color online) Height fluctuations around the mean value for each reconstructed surface profile in Fig. 4. Units are pixels (horizontal and vertical axes), and $\mu \mathrm{m}$ (height).

resultant speckle introduces random spatially varying phase shifts in the interferogram. Unambiguous depth information is still provided on a pixelwise basis, however, from the frequency of the intensity modulation with respect to wavenumber. In effect the system provides a 2D array of rangefinders, with an acquisition time that can be made arbitrarily small given a pulsed white light source of sufficient intensity, and a measurement precision on rough metal surfaces of around $0.5 \mu \mathrm{m}$. Potential applications include areal roughness measurement and probes for coordinate measurement machines where rapid data acquisition in noncooperative environments is essential. Future development will focus on the design of an optical system to distribute the images across the full photodetector array, and hence make more efficient use of the available pixels.

The work reported in this paper is part of $\mathrm{T}$. Widjanarko's Ph.D. research at Loughborough University. He is grateful to the Islamic Development Bank scholarship programme for funding his Ph.D. studentship.

\section{References}

1. T. Dresel, G. Häusler, and H. Venzke, Appl. Opt. 31, 919 (1992).

2. M. Takeda and H. Yamamoto, Appl. Opt. 33, 7829 (1994).

3. S. Kuwamura and I. Yamaguchi, Appl. Opt. 36, 4473 (1997).

4. J. M. Huntley, T. Widjanarko, and P. D. Ruiz, Meas. Sci. Technol. 21, 075304 (2010). 